\title{
Les prisons politiques chez Marlowe et Shakespeare
}

\section{Pierre Sahel}

Marie-Thérèse Jones-Davies (éd.)

\section{(2) OpenEdition \\ Journals}

Édition électronique

URL : http://journals.openedition.org/shakespeare/128

DOI : $10.4000 /$ shakespeare. 128

ISSN : 2271-6424

Éditeur

Société Française Shakespeare

Édition imprimée

Date de publication : 1 novembre 1980

Pagination : 99-112

Référence électronique

Pierre Sahel, «Les prisons politiques chez Marlowe et Shakespeare », Actes des congrès de la Société française Shakespeare [En ligne], 2 I 1980, mis en ligne le 01 novembre 2007, consulté le 06 mai 2019. URL : http://journals.openedition.org/shakespeare/128; DOI : 10.4000/shakespeare.128 


\section{SOCIÉTÉ FRANC̣AISE SHAKESPEARE}

\section{ACTES DU CONGRĖS 1980}

DIRECTEUR DE LA PUBLICATION

M.T. Jones - Davies

JEAN TOUZOT Libraire - Editeur 38 , rue Saint-Sulpice 75278 PARIS CEDEX 061981 


\section{LES PRISONS POLITIQUES CHEZ MARLOWE ET SHAKESPEARE}

En étudiant les dramatisations des prisons politiques marloviennes et shakespeariennes, qui sont souvent - je le montrerai - diamétralement opposées ${ }^{1}$, je me suis demandé comment le monde de la prison, et singulièrement de la prison politique, pouvait être compatible avec le monde dramatique.

Alors, en effet, que le dramatique vit de gestes, de mouvements, de paroles échangées, qu'il engendre parfois des situations d'une liberté hénétique! sans rapport avec l'existence ordinaire, l'univers carcéral se définit par la restriction des possibles du monde extérieur. En prison règnent l'ennui, l'attente, la solitude, au fil de jours scandés par des activités que leur répétition rend haïssables ${ }^{2}$. Des témoignages multiples de détenus ${ }^{3}$ indiquent que la prison politique n'est pas moins que la prison de droit commun un monde sans vie. Bien plus, si celle-ci peut vouloir sanctionner un forfait ou rééduquer un malfaiteur, celle-là se contente d'engloutir son hôte. Elle le met hors d'état non de nuire, mais d'agir,le liquide physiquement parfois plus sûrement que la mort - toujours susceptible de donner au condamné l'auréole du martyre. Du reste, l'analogie entre la prison et la mort constitue, sous la plume de détenus politiques, un leitmotif lancinant 4 . Dostoievski n'appela-t-il pas son témoignage sur le bagne Souvenirs de la maison des morts?

Avec le recul du temps, on pourrait bien trouver un certain pittoresque à la Fleet ou à la Clink. Les prisons des XVIè et XVIIè siècles ne sont pas,en effet, coupées du monde extérieur, et les appels des détenus pauvres qui, à travers les barreaux, mendient quelque nourriture aux passants comptent parmi les bruits familiers du Londres de cette époque. L'argent joue alors en prison un rôle insolite ${ }^{5}$, et les riches détenus peuvent non seulement aller et venir dans l'enceinte de l'établissement qui les héberge mais, accompagnés du gardien ou de la cuisinière des lieux, ils en sortent parfois pour faire des courses, voire pour boire du sack à la taverne ${ }^{6}$. Nous possédons quelques témoignages sur l'étonnante liberté dont jouissaient certains prisonniers politiques même lorsqu'ils n'étaient pas des personnages de 
marque ${ }^{7}$. Mais, certes, en général, l'espace carcéral de la Renaissance est, comme celui d'autres époques, un lieu morne où le prisonnier mène une existence végétative et souffre. Lorsqu'il s'agit d'arracher des aveux ou des révélations aux opposants politiques, les tortures y sont employées, que des instructions officielles, par exemple après le complot des Poudres, recommandent lentes et régulières ${ }^{8}$. Dans son Humble supplique à Sa Majesté, Robert Southwell décrit la puanteur qui y règne et les ravages qu'y cause la vermine. La dureté des gardiens est légendaire. La métaphore tombe/ cachot que j'ai signalée est fréquente dans la prose de ceux qui ont été incarcérés. Pour John Earle, la prison, c'est la tombe où l'on enferme les vivants; Pluton y tient sa' cour $^{9}$. Pour Dekker, elle n'est pas proche de l'enfer; elle est l'enfer même ${ }^{10}$. Pour Geoffrey Mynshul, dès qu'on atteint les grilles d'une prison, on pénètre en enfer ${ }^{11}$.

Le problème esthétique de la transposition réaliste de la prison, monde terne et sans vie, se pose donc autant aux dramaturges renaissants qu'aux dramaturges plus proches de nous dans le temps. Au demeurant, il n'existe' pas qu'au théâtre. Songez au cinéma, songez aux feuilletons télévisés : la plupart du temps, la prison n'y est, comme telle, montrée qu'au moment où le héros prépare son évasion, où la mutinerie se déclenche ..., car seuls de tels incidents peuvent intéresser les spectateurs. Songez aux quelques exemples littéraires célèbres dont un épisode se situe en prison ( $L a$ chartreuse de Parme de Stendhal, Résurrection de Tolstoï...): la prison y est toujours traitée marginalement; elle n'est jamais au centre de l'intrigue. Il faudrait à l'homme de lettres un singulier talent pour décrire par le menu la vie monotone du prisonnier, un talent peut-être proche de celui de Dino Buzzati, dont Le désert des Tartares n'est pas, dans son atmosphère, sans rapport avec le monde carcéral.

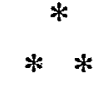

Souvent - on n'en sera donc pas surpris - la dramatisation de la prison, et particulièrement de la prison politique n'est qu'une frange étroite du théâtre de Shakespeare comme de celui de Marlowe. Certes, une quinzaine de pièces shakespeariennes traitent de près ou de loin de la prison et 
de prisonniers; certes, de très longues scènes de Mesure pour mesure sont des scènes de prison; certes, de mémorables passages des Deux nobles cousins ou du Roi Lear transfigurent la prison. Mais la présentation précise de la prison politique offre une difficulté spécifique à ajouter à celle qui consiste à se référer à un espace normalement morne et vide : comme le chef politique responsable d'une incarcération se trouve être, dans bien des cas, le maître du jeu de la pièce à laquelle il appartient, il n'a aucune raison d'extraire du cachot celui qu'il a fait, comme on dit, mettre au secret - et donc de lui permettre d'apparaître sur la scène.

Ainsi, lorsque dans la troisième «Partie» du drame qui porte son nom, Henri VI est emprisonné, il disparait de la scène. Arrêté au début de l'Acte III au nom du roi d'York que la fortune des armes et une légitimité rivale ont porté au pouvoir, il ne reparaît qu'à la fin de l'Acte IV, lorsque les partisans de la Rose rouge lui ont rendu son trône; arrêté à nouveau au début de l'Acte $V$, il disparaît encore, jusqu'au «souper sanglant» que Richard de Gloucester lui impose de partager' à la Tour de Londres, à l'extrême fin de la pièce. Dans Antoine et Cléopâtre, l'emprisonnement de Lépide, étape poutant importante de la marche qui conduit César à devenir l'unique pilier du monde romain, n'est mentionné qu'en deux lignes ${ }^{12}$. Dans Henri VIII, victime des intrigues de Wolsey, le duc de Buckingham est arrêté à l'acte I; il ne remonte sur scène à l'Acte suivant qu'entre deux haies de hallebardiers avant d'être conduit au billot. Le sort logique d'un prisonnier politique shakespearien pourrait donc sembler être ou l'absence ou la mort. L'analogie entre la mort et le cachot existe d'ailleurs dans Shapeare ${ }^{13}$. Or la mort politique n'est pas, par nature, plus dramatique que l'emprisonnement politique, car la compétence des spadassins envoyés dépêcher une victime politique s'applique davantage à un concept ou à un objet qu'à un homme. Lorsque le rouage politique est parfait, le dramatique est totalement absent de la scène. Dans Richard III, Richard procède à une exécution massive; les condamnés sont des parents de la reine et constituent un clan politique hostile au pouvoir du Protecteur. Aucun ressort dramatique n'est alors pressé. La scène est même quelque peu comique par le désir exprimé par Ratcliff, l'homme de main de Richard, de ne pas s'encombrer trop longtemps d'un matériel humain 
à détruire. «Dépêchons», dit-il aux condamnés sur le chemin de l'échafaud, «la limite de votre vie est franchie» ${ }^{14}$; et «Hâtons-nous. L'heure de votre mort est déjà passée ${ }^{15}$;

puis, à la scène suivante, lorsque c'est au tour de Hastings d'être exécuté :

Allons, allons ! Dépêchez ! Le duc voudrait dîner; faites une courte confession. Il lui tarde de voir votre tête. ${ }^{16}$

C'est le point de vue du fonctionnaire diligent, du croquemort pressé - ou encore du fossoyeur de Hamlet qui provoque l'étonnement du prince parce qu'il chante et boit de la bière en creusant ses tombes.

Dans Marlowe comme dans Shakespeare, les apparitions de prisonniers sont furtives. Dans 1 Tamerlan, le plus «pittoresque» de ces prisonniers - encore s'agit-il plus d'un prisonnier de guerre que d'un prisonnier politique -.. est Bajazet, qui apparaît sur scène dans une cage (IV. ii) contre laquelle il finira par se fracasser le crâne (V.ìi). Dans $2 \mathrm{Ta}$ merlan, une brève scène de prison (I.iii) voit Callapine corrompre pour s'évader son geôlier, Almeda. Dans Le Docteur Faust, Bruno, l'anti-pape, traverse la scène chargé de chaînes (III. ì); il est,lui, prisonnier politique car il a été choisi par l'Empereur pour contrecarrer le pouvoir papal; au cours de sa brève captivité - puisque Faust et Méphistophélès ne tardent pas à favoriser sa fuite - il ne fait que servir de tabouret au pape. Dans Edouard II, quelques vers seulement sont consacrés à l'arrestation de l'évêque de Coventry, victime de la vengeance politique du roi et de son mignon ${ }^{17}$. Victorieux au début de la guerre civile, Edouard envoie à la mort les barons tombés entre ses mains, et ne leur accorde qu'une oraison funèbre sarcastique de deux vers (IV.iit, 12-13). Lorsque le vent a tourné, que le parti royal, à son tour, est défait, Rice Ap Howell écourte les ultimes discours des fidèles d'Edouard. "Allons, allons !», dit-il à Baldock et à Spencer qui philosophent sur leur mort imminente «gardez ces sermons pour l'endroit convenable!"

L'évanescence de ces cohortes de prisonniers politiques me fait penser que Marlowe et Shakespeare étaient éminement conscients de la faible compatibilité du monde carcéral et du monde dramatique. Je crois même que Shakespeare, au moins, s'est explicitement interrogé sur les 
problèmes que pose la mise en théâtre de la prison politique. Son interrogation, il faut le remarquer, est la même que celle du détenu. Car que fait l'homme enfermé aux prises avec l'ennui, la solitude ...? Il monologue. C'est ce qu'a fait, par exemple, Altiero Spinelli, militant politique italien longtemps emprisonné, qui livre ses méditations dans un ouvrage intitulé Le long monologue. C'est ce que fait, bien sûr, Richard II dans un des plus longs monologues de l'œuvre' de Shakespeare. L'obsession première du prisonnier politique, c'est le temps. Témoin cette réflexion de MartinChauffier :

Le temps, pour nous, ne passait pas; c'est nous qui passions, toujours plus las, sur ces jours immobiles; remplis de souffrances, d'inquiétudes ou de monotonie, mais immobiles (...). C'était vraiment du temps perdu et qui nous perdait avec lui. ${ }^{19}$

Que l'on me pardonne cette unique mention de l'obsession du temps. Seul le temps qui m'est imparti pour cette communication - je le dis sans ironie -.. m'empêche de multiplier mes références à cette préoccupation si fréquente dans les témoignages de prisonniers politiques ${ }^{20}$ dont Richard II semble avoir été le prototype, lui qui constate :

J'ai abusé du temps, et, à présent, le temps abuse de moi; car, à présent, le temps fait de moi son horloge 21

Plongé dans la solitude, le détenu politique cherche à meubler et son temps et son espace clos. Adolf Hoffmeister apostrophait les mur et la chaise de son cachot. Il s'inventait un compagnon avec lequel dialoguer, un peu comme Boris Vildé qui, lui, se mettait à dialoguer avec lui-même, s'inventant, comme il l'écrit, «deux moi, authentiques l'un et l'autre». Il poursuit :

«Il est difficile de les définir avec précision; aussi, je les désigne tout simplement par moi 1 et moi $2 .{ }^{22}$

Or c'est le même effort, pour résister à la solitude et rompre la monotonie, qu'entreprend Richard II en des mots où l'interprétation métadramatique aurait beau jeu de percevoir la voix de Shakespeare :

J'ai étudié comment je pourrais comparer la prison où je vis avec le monde; mais, comme le monde est populeux et qu'ici il n'y a d'autre 
créature que moi, je n'en ai pas trouvé moyen. Pourtant, forgeons un rapprochement (...). Ainsi, je joue à moi seul bien des personnages (...) par moments, je suis roi (...), puis me voilà mendiant ${ }^{23}$. Comme son créateur, pourrait-on dire, Richard éprouve bien des difficultés (《j’ai étudié», «forgeons», «je n'ai pas trouvé») à peupler la scène de sa prison, bien des difficultés non à expliquer quoi que ce soit, mais à faire quelque chose $^{24}$. Méditer ainsi sur la prison politique, c'est déjà la dramatiser, puisque le personnage exploite jusqu'à l'extrême les possibilités théâtrales de sa pitoyable situation ${ }^{25}$,donnant voix, par exemple, à ses pensées et les interpellant.

S'il est vrai qu'en même temps que son personnage Shakespeare se pose et les questions d'un prisonnier et les les problèmes d'un dramaturge occupé à décrire la prison, il doit les résoudre en parvenant, comme Marlowe, à susciter l'intérêt, la passion, la sympathie ... des spectateurs pour des hommes que d'autres personnages, les vainqueurs du jeu politique des drames, ont résolu de ne plus voir et de ne plus entendre. Aux yeux des vainqueurs, un Edouard,un Richard ou un Clarence sont des principes d'opposition à écarter de la Cité, voire à supprimer, car c'est au nom de ces hommes que la révolte gronde ou peut se lever ou encore que la résistance peut s'organiser. A nos yeux, au contraire, ces prisonniers doivent demeurer ou devenir des êtres dramatiques vivants. Les efforts de Shakespeare et de Marlowe doivent aboutir à opérer une dissociation radicale entre les chefs politiques victorieux et les spectateurs de leurs drames: pour les premiers, la mise à l'écart du prisonnier est une froide nécessité, sa mort la solution finale proposée au problème qu'il continue de poser; pour les seconds, le prisonnier doit être un personnage digne d'intérêt et sa mort une force scandaleuse.

$$
\begin{gathered}
* \\
* *
\end{gathered}
$$

Pourtant la mise à mort du principal prisonnier politique du théâtre marlovien est, techniquement, le chefd'œuvre de l'assassinat politique. Parce que son gouvernement ne jouit pas de la moindre légitimité, Mortimer tient à ce que le meurtre du roi déchu soit perpétré en secret et 
à ce que sa mort semble naturelle. L'ordre d'exécution est un modèle d'ambiguité; rédigé en latin et non ponctué, il peut vouloir dire : «craignez de tuer le roi» ou «tuez le roi» (V. iv. 6-12). Edouard est mis à mort selon un processus voisin du supplice du pal : tandis que les aides de l'exécuteur maintiennent la victime sous une table qu'ils piétinent, «mais pas trop fort, pour ne pas blesser le corps» ${ }^{26}$, le tueur déchire les entrailles du supplicié au moyen d'un tournebroche rougi à blanc enfoncé à partir de l'anus. Ainsi ne sera visible aucune trace de violence. "C'est de la belle besogne» ${ }^{27}$, conclut le tueur. C'était de la «belle besogne» qu'avait exigée Mortimer ${ }^{28}$. Mais cette mort propre, cette réussite technique parfaite, soulève notre horreur, et c'est l'émotion violente de ce témoin partial, le spectateur, qui empêche la mise à mort d'être la simple transformation, conduite selon un mode opératoire quasi scientifique, d'un déchet politique en un déchet physique ${ }^{29}$. Or la partialité horrifiée du spectateur en faveur d'Edouard ne naît pas de la simple pitié pour la victime assassinée, pitié qui pourrait fort bien ne pas s'éveiller en des sensibilités autres que modernes. Elle est l'aboutissement de la dramatisation que Marlowe a su mener à bien de l'emprisonnement du roi. Marlowe est parvenu à nous intéresser - plus, à nous river émotionnellement - au monde où est retenu son personnage en le dépeignant comme un lieu de tortures distillées au fil des jours. De ses sources, il a choisi et réuni les détails les plus horrifiants. La dernière prison d'Edouard est une fosse où se déversent les égoûts du château de Berkeley ${ }^{30}$. Le malheureux est donc plongé à mi-corps dans un bourbier malsain. Son régime alimentaire se réduit au pain et à l'eau (V. v. 61), et la viande qui lui est jetée par la porte entrebaillée (V.v. 8) améliore peut-être son ordinaire. Ses vêtements sont en lambeaux (V.v. 66). Demande-t-il de l'eau pour se laver? On lui impose de l'eau boueuse ${ }^{31}$, on le rase de force avec de l'eau du ruisseau, si bien que cette toilette brutale ressemble à un attentat contre sa vie (V.iii. 30-31). Tout, au cours de sa captivité, est avilissant, depuis son arrestation, conduite avec des Welsh hooks, instruments qui n'ont rien à voir avec les armes de guerre ${ }^{32}$, jusqu'au supplice du pal que j'ai décrit. La cruauté des conditions de sa détention se double de la cruauté mentale qui anime ses tortionnaires ${ }^{33}$. On bat le tambour jour et nuit pour l'empê- 
cher de dormir (V. v. 61-62). On le change sans cesse de lieu de détention. Comme un gibier que l'on traque par plaisir plutôt que pour l'abattre, on lui fait craindre le pire, mais on le rassure aussi : d'accord avec son amant pour torturer son époux, la reine Isabelle fait parvenir à Edouard une bague en témoignage de tendresse (V.ii .72); Lightborn, qui se targue pourtant de pouvoir mener ses assassinats avec la rigueur d'un homme de métier (V.iv . 29-37), fait mine de réconforter le prisonnier et d'avoir le cœur brisé devant tant de misères (V. v. 42,56,70). La condition présente du prisonnier offre un contraste saisissant avec sa condition passée élevée, avec les codes auxquels il pouvait (ou aurait $\mathrm{pu}$ ) se référer jadis': lui qui est dans la fange et se nourrit de pain n'oublie pas qu'il fut roi (V.v.61), ne peut oublier qu'un jour il caracolait selon les rites chevaleresques pour l'amour de sa belle (V . v. 67-69) - de celle qui devait devenir la reine Isabelle! On pourrait presque soupçonner, tant est grande la propension du prisonnier à évoquer lyriquement le passé, qu'il éprouve à se voir souffrir un plaisir morbide qui viendrait à point nommé renforcer le «sadisme» de Mortimer, d'Isabelle ou de Lighborn. Témoin ces gestes, pathétiques dans leur courtoisie : son envoi à Isabelle d'un mouchoir trempé de larmes (V. I. 118), son offre d'une perle à Lightborn (V.v. 83). Pourtant la self-pity d'Edouard n'exagère pas l'enfer du cachot, car l'étonnement de Matrevis et de Gurney, les geôliers au cœur taillé dans un rocher du Caucase ${ }^{34}$, devant la survie du roi (V. v. 1-15) confirme l'inhumanité de ses conditions de vie.

Marlowe dramatise ainsi la prison d'Edouard II comme l'antre de misère (the cave of care) où agonise l'ex-roi. Il maintient ses spectateurs dans la fascination de l'horreur et d'un mal infligé au-delà des bornes de la raison et, certes, au-delà de toute nécessité politique. Cette fascinante et cruelle dramatisation, qui conduit à juger la pièce comme l'étude d'un mal inhumain pratiqué par des hommes ${ }^{35}$, captive le spectateur ... dont le point de vue est celui du captif, non certes celui du vainqueur politique.

Shakespeare parvient à nous intéresser à l'univers de ses prisons politiques par une dramatisation très différente. C'est à peine si, dans Richard III, existe une forme d'《ironie dramatique» qui s'apparente au «sadisme» de Lightborn dans la prétention affichée par Richard de Gloucester de 
venir en aide au frère qu'il a lui-même fait jeter en prison et qu'il va lui-même faire exécuter (I I ́ . 107-116). Mais dès que les tueurs approchent de Clarence endormi, le scrupule et la pitié tentent d'investir le cachot, si bien que le Premier Assassin doit inciter son complice à remettre sa conscience dans la relation d'échange établie avec Richard de Gloucester : la mort à donner en échange de l'or reçu. Persuadé pour un temps, le Deuxième Assassin se lance dans une diatribe contre ladite conscience, qu'il remet dans la bourse du duc :

Elle ruine quiconque la garde; elle a été chassée des villes et des cités comme un être dangereux, et tout homme qui entend vivre à l'aise tâche de ne se fier qu'à lui-même et de vivre sans elle ${ }^{36}$.

Ces paroles constituent un véritable développement d'économie politique. En témoignent les termes commerciaux : «ruine», «garde», «vivre à l'aise»; les villes sont perçues comme les carrefours, les lieux d'échanges, d'où la conscience, qui n'est pas un bien économique et qui freine les transactions malhonnêtes, a été expulsée. Le Deuxième Assassin voudrait l'expulser de lui-même afin de devenir pur objet. Mais la paradoxale fluidité de la conscience est telle que, chassée du monde de la Cité, elle reparaît au sein du cachot microcosmique de la Tour : le Deuxième Assassin finira par renoncer à sa récompense - regagnant peut-être ainsi sa conscience ! Dans Le roi Jean, Hubert se veut insensible, inflexible. Il présente au petit prince Arthur, rival dynastique--bien involontaire-du roi,l'ordre qui le condamme. Il active les exécuteurs, fait apprêter des fers rougis à blanc. Pourtant ses nombreux apartés révèlent ses scrupules. Bientôt, son humanité déborde et fait : craquer le masque officieux d'impassibilité qu'il s'efforçait de porter devant sa jeune victime. Ce qui constitue donc le ressort dramatique des scènes du château prison ou de la Tour, c'est la rupture de la dialectique selon laquelle le bourreau se considère comme un instrument et considère sa victime comme un objet à détruire. Hubert redoute cette rupture, et veut empêcher que s'établisse une dialectique humaine entre lui et Arthur :

$\mathrm{Si}$ je lui parle, il va, avec son innocent babil, éveiller ma pitié, tout enterrée qu'elle est.

Donc, soyons brusque, et dépêchons. ${ }^{37}$

Or Arthur parle et, par un jeu verbal désespéré, signale sa jeunesse, sa tendresse, ses émotions au tueur qui va finir par 
l'épargner. Or Clarence s'éveille et ne traite pas les deux spadassins comme de simples exécutants ou comme les instruments d'un pouvoir inflexible, mais comme des êtres humains ayant la faculté de ne pas donner la mort, comme ils avaient celle de la donner. Même si, alors, les envoyés de 'Richard de Gloucester essaient de justifier l'acte qu'ils ont à commettre, en rappelant à Clarence ses crimes passés, ils se laissent, du moins, glisser à réintroduire les notions morales qu'ils avaient tenté d'expulser d'eux-mêmes. Ils outrepassent ainsi leur médiocre fonction brutale, radicale, définitive de criminels à gages. Le dialogue s'établissant entre tueurs et victimes, la communication fait des assassins de Clarence des juges, parfois émus; l'échange, plus humain, instaure une sorte de sympathie, même éphémère.

Je reviens, avant de terminer, sur la prison de Richard II. Encore une fois pour intéresser le spectateur en humanisant le monde de pierres contre lesquelles, dit Richard, les ongles de l'homme enfermé ne peuvent rien (V.v.. 19-21), Shakespeare adoucit les derniers moments du roi.ll y a d'abord la visite du palefrenier venu, à ses risques et périls, saluer son ancien maître du titre de prince royal - en échange de quoi, l'autre l'élève avec humour à la pairie des cachots (V. v .67-68). Il y a aussi cette mauvaise musique anonyme qui monte vers l'antre de la majesté déchue comme un message de sympathie et que le prisonnier perçoit avec reconnaissance :

Béni soit le noble cœur qui me fait don de sa musique, car c'est un signe d'amour. ${ }^{38}$

Ainsi, pour aviver sa peintre du monde de la prison politique Shakespeare en oublie les aspects les plus froids au profit de points. de vue moraux et d'accents dramatiques. A la souffrance de la réclusion comme au scandale de la mort, il oppose le miracle d'un amour insolite ou d'une sympathie paradoxale qui lie les hommes entre eux, qui leur fait aussi découvrir leur commune humanité dans des situations d'antagonisme ${ }^{39}$. 


\section{NOTES}

1. Le contraste que je présente ci-après est à verser au dossier - déjà épais - sur les rapports, les oppositions et les affinités entre Shakespeare et Marlowe. Voir l'important esssai de Nicholas Brooke, «Marlowe as Provocative Agent in Shakespeare's Early Plays", Shakespeare Survey XIV (1961), pp.34-44, et la plus récente étude sur le sujet, Muriel C. Bradbook, «Shakespeare's Recollections of Marlowe», in Shakespeare's Styles : Essays in Honour of Kenneth Muir (Cambridge U.P., 1980), pp. 191-204.

2. Voir Anon, «le monde des prisons», Esprit 225 (Avril 1955), en particulier pp. 505-506.

3. Voir Bernadette Morand, Les écrits des prisonniers politiques (Paris, 1976), p. 102, p. 119, p. $79 \ldots$

4. Ibid., p. 173.

5. Nous le savons en particulier grâce aux écrits d'un ancien gardien de prison accusé d'avoir escroqué les prisonniers qui lui étaient confiés : tout se monnaie alors en prison - confort, égards, liberté de ne pas porter les fers... Voir Alexander Harris, The Economy of the Fleet : Or an Apologetical Answer (...) unto Nineteen Articles ..., ed. Augustus Jessopp, Camden Soc., NS, XXV. (1879).

6. Geoffrey Mynshul, Essays and Characters of a Prison and Prisonners (1618), pp. 52-53.

7. Dans un récit écrit en latin, plus tard traduit et publié sous le titre de The Autobiography of an Elizabethan (tr. Philip Caraman, London, 1951), John Gerard, père jésuite emprisonné, décrit un trafic de clefs de cellule, un réseau de communications avec l'extérieur... L'auteur put dans sa prison recevoir plusieurs confessions, convertir plus d'un détenu et même célébrer la messe interdite sur l'étendue du territoire anglais. 11 écrit (p. 78) : "Provided only I could have stayed on in this prison, I should never have wanted to have my liberty again in England.»

8. Calendar of State Papers, Domestic Series, 1603.1610, vol. XVI (London, 1857), p. $258:$ : (...) that the prisoners be confined apart, in darkness and examined by torch-light, and that the tortures be slow and at intervals, as being most effectual.»

9. Microcosmography, ed. Harold Osborne (1933), p. 26.

10. Cité par E.D. Pendry, Elizabethan Prison and Prison Scenes (Salzburg, 1974), p. 281.

11. Essays, p.49.

12. III. v.10-12. Fdition des œuvres de Shakespeare utilisée : Peter Alexander (London, 1951 ).

13. Le portier de Macbeth se désigne comme le geôlier de l'enfer. Au dernier Acte de Hamlet, le prince de Danemark, sentant le froid de la mort l'envahir, compare la mort à un gendarme féroce venu l'arrêter. Dans Cy'mbeline 
un échange nourri de plaisanteries sur la mort à lieu entre Posthumus et ses gardiens.

14. Make haste; the hour of death is expiate(III.iii.23).

15. Dispatch; the limit of your lives is out (III .iii.8).

16. Come, come, dispatch; the Duke would be at dinner. Make a short shrift; he longs to see your head. (III.iv.96-97)

17. I 1 187-198. Edition utilisée : Edward $I I$, H.B. Charlton (London, 1933).

18. Come, come keep these preachments till you come to the place appointed (IV.vi, 11 2-1 13 ).

19. Cité par Bernadette Morand, p. 124.

20. Voir Bernadette Morand, p. 102, p. $123 \ldots$

21. I wasted time, and now doth time waste me.

For now hath time made me his numb'ring clock. (V.v.49-50)

22. Cité par Bernadette Morand, p. 134.

23. I have been studying how I may compare

This prison where I live unto the world;

And, for because the world is populous

And here is not a creature but myself,

I cannot do it. Yet I'll hammer it out.

Thus play $I$ in one person many people.

Sometimes am I king; ....

Then treasons make me wish myself a beggar

And so I am.

$$
(\mathrm{V}, \mathrm{v}, 1-\ldots .34)
$$

p. 153.

24. Wolfgang Clemen, Shakespeare's Dramatic Art (London, 1972),

25. ibid., p. 154.

26. But not too hard, lest that you bruise his body. ( $V, v, 112)$

27. Was it not bravely done? (V.v.115)

28. Do it bravely. (V. v. 28)

29. Nicholas Brooke («Marlowe the Dramatist», Stratford-uponA von Studies, IX (1966), pp. 103-104) s'interroge sur «l'intolérable morale» du meurtre d'Edouard.

30. Holinshed décrivait le lieu de détention comme une pièce située au-dessus d'un dépotoir. 
31. Ce n'est pas dans Holinshed mais dans Stow (Annals of England, 1580) que Marlowe a trouvé mention de cet te toilette.

32. Michael J. Warren, "Welsh Hooks in Edward II», Notes and Queries NS, XXV, 2 (1978), pp. 109-110.

33. Mortimer ordonne que l'on parle rudement au prisonnier et qu'on lui refuse toute consolation (V. ii. 65-67). Matrevis parle d'assaillir l'esprit du roi $(\mathrm{V}, \mathrm{v} .12)$.

34. Thy heart, were it as Gurney's is, Or as Matrevis', hewn from the Caucasus ... (V. v., 52-53)

35 R.A. Watson, "Edward II : A Study in Evil», Durham University Journal, LXVIII, NS (June1976), pp. 162-167.

36. It beggars any man that keeps it. It is turn'd out of towns and cities for a dangerous thing:and every man that means to live well endeavours to trust to himself and live without it. (I. ív. 138-142)

37. If I talk to him, with his innocent prate He will awake my mercy, which lies dead. Therefore I will be sudden and dispatch. (IV , i , 25-27)

38. Yet blessing on his heart that gives it me ! For 'tis a sign of love. (V.v.64-65)

39. J'espère ne pas avoir borné ma comparaison de Shakespeare et de Marlowe au domaine des idées mais avoir suivi cette recommandation de Wolfgang Clemen : "In order to show why two plays are so different from one another and evoke such varying responses, we must describe their «physiogno. my», their dramatic rhythm.» («Shakespeare and Marlowe», Proceedings of the World Shakespeare Congress, Vancouver (1971), Shakespeare 1971, p. 125). 